\title{
Enhancement of Ion Intensity in Time-of-Flight Secondary-Ionization Mass Spectrometry
}

\author{
Anthony J. Nicola, David C. Muddiman, and David M. Hercules \\ Department of Chemistry, Vanderbilt University, Nashville, Tennessee, USA
}

\begin{abstract}
Enhancement of ion intensity in static secondary-ionization mass spectrometry (SIMS) has been achieved by using a matrix-assisted sample preparation technique. Previous investigations of polymers and biomolecules by SIMS indicated that secondary-ion (SI) yield is dependent on substrate coverage. Recently we discovered a sample preparation technique that enhanced the SI yicld of cyclosporin A (CSA) in an allograft patient sample and neat samples of CSA $(1202 \mathrm{u})$ and polystyrene $\left(M_{\mathrm{w}}=2650 \mathrm{u}\right)$. The preparation technique involves deposition of a submonolayer of cocaine hydrochloride $(5 \mu \mathrm{L}$ of a $20-\mu \mathrm{g} / \mathrm{mL} \mathrm{MeOH}$ solution) on an etched silver substrate, solvent evaporation, and subsequent deposition of the analyte. This preparation method resulted in $\sim 300 \%$ increase in the SI yield of CsA and polystyrene when deposited from neat solutions. The original discovery was observed when a blood extract that contained CsA was deposited on an etched Ag substrate that had been soaking in a dilute cocaine solution for $\sim 2$ months. In these initial experiments, the SI yield of CsA was enhanced by over 1 order of magnitude. (I Am Soc Mass Spectrom 1996, 7, $467-472)$
\end{abstract}

S tatic secondary-ionization mass spectrometry (SIMS) has been used to investigate a wide range of analytical systems. To date, SIMS research has focused mainly on qualitative analysis of polymers [1-5], additives [6], adhesives and coatings [7], semiconductors [8], and biological materials [9]. Analysis of polymers and biomolecules by SIMS typically is performed by deposition on a chemically etched silver substrate. However, due to the low penetration depth of the primary ion beam $(\sim 10 \AA)$, coverage of the $\mathrm{Ag}$ substrate directly influences the secondary-ion yicld in SIMS [10]. Higher surface coverages are thought to decrease the effectiveness of the collisional cascade produced by the impinging primary ions.

Time-of-flight SIMS (TOF-SIMS) is a valuable method for characterization of several polymeric [1-5] compounds, as well as analysis of biological [11-15] materials from physiological media. Analysis of such samples in our laboratory has shown that the secondary-ion (SI) yield of both types of materials depends on surface coverage $[10,12]$. In the analysis of polymeric materials, iterative experiments can be carried out to optimize ion intensity. However, the variability in extracts of biological fluids is difficult to control because they do not produce "neat samples" and the extent of contamination from endogenous ma-

Address reprint requests to Professor David M. Hercules, Depart ment of Chemistry, Vanderbilt University, Nashville, TN 37235.

* Present address: Battelle Memorial Institute, Pacific Northwest National Laboratories, Environmental Molecular Science Laboratory, Battelle Boulevard Box 999, Richland, WA 99352. terial varies from extract to extract. Optimization of each extract is hindered by limited sample size and the time required for analysis; the latter is especially important if TOF-SIMS is to be used for routine biomedical analysis.

In the early 1980s, a novel sample preparation method for static SIMS was introduced by Liu et al. [16]. This method involved premixing neat solutions of simple sugars, mono-and polypeptides, nucleotides, and vitamins with a saturated solution of ammonium chloride. This mixture was then deposited onto a variety of metal substrates (i.e., $\mathrm{Ag}, \mathrm{Cu}, \mathrm{Sn}, \mathrm{Pt}$, and $\mathrm{Fe}$ ). Good molecular ion signals, which include $[\mathrm{M}+\mathrm{H}]^{+}$, $[\mathrm{M}+\mathrm{Na}]^{+}$, and $[\mathrm{M}+\text { metal }]^{+}$(only with $\mathrm{Cu}$ and $\mathrm{Ag}$ ), were reported when the ammonium chloride matrix was used [16]; however, no molecular ion signal was observed without the modified preparation technique. Further work with this sample preparation method, which was termed "matrix-assisted SIMS," included analysis of low molecular weight organic ammonium compounds and pyridinium salts from neat solutions. Enhanced molecular ion intensity was observed when an excess of ammonium chloride was added to the analyte solution, compared to analysis without the ammonium chloride matrix $[17,18]$; the intensity ratio of intact cation to fragment was increased by a factor of $\sim 5$ when the matrix was used [18]. Unger et al. [17] reported a detection limit of lower than $50 \mathrm{ng}$ of 1-adamantanamine from a solution diluted 1:200 with ammonium chloride and Busch et al. [18] achieved a detection limit of $0.5 \mathrm{ng}$ of a quaternary ammonium salt from a solution diluted 1:50000. 
In addition to an increase in molecular ion intensity, use of the ammonium chloride matrix also yielded a significant reduction in the observed fragmentation of the analyte [16-18]. Busch and co-workers proposed a mechanism where large "clusters" of ions are sputtered during bombardment by the primary ions. These clusters contain analyte ions surrounded by, or solvated by, the matrix ions. Reduced fragmentation was explained to be a result of a desolvation process, which caused a relaxation of the internal energy responsible for fragmentation of the analyte [18]. Unger et al. [17] postulated that the increase in molecular ion intensity was a result of proton donation from the ammonium ions in the matrix to the analyte. A reduction in both fragmentation and adduct formation could be explained by elimination of intermolecular reactions (among the analyte ions) due to dilution by the matrix [17].

This article reports the discovery of a similar sample preparation technique. Our sample preparation method is very useful for analyses of real biological samples (i.e., blood extracts). Use of this method increases the SI yield of neat biological and polymer samples as well. Our initial discovery is described in addition to experiments conducted to reproduce this initial observation. Possible mechanisms of the effect, which focus on surface-related aspects, are considered in the context of the mechanisms proposed in the initial work. Future experiments also are proposed to further elucidate the mechanism. Additionally, practical applications for analysis of polymers and biological materials by using our sample preparation technique are presented.

\section{EXPERIMENTAL}

\section{Materials}

Cyclosporin A (CsA; $1202 \mathrm{u}$ ) was obtained from Sandoz Corporation (Basel, Switzerland). Polystyrene was obtained from Polymer Laboratories (Amherst, MA), with an average molecular weight $\left(M_{\mathrm{w}}\right)$ of $2650 \mathrm{u}$ and a polydispersity index $\left(M_{\mathrm{w}} / M_{\mathrm{n}}\right)$ of 1.05 . Cocaine hydrochloride was purchased from Sigma Chemical Co. (St. Louis, MO) as a pure powder and dissolved in high-performance liquid chromatography grade methanol (Fisher Scientific, Fair Lawn, NJ). Puratronic (99.9985\%) silver foil ( $0.25 \mathrm{~mm}$ thick) was obtained from Alfa and was etched in trace-metal grade $\mathrm{HNO}_{3}$ $(20 \mathrm{vol} \%)$ purchased from Fisher Scientific. The geometrical surface area of all silver substrates used was $150 \mathrm{~mm}^{2}$. All materials were used without further purification.

\section{Instrumentation}

All experiments were performed with a TOF-SIMS mass spectrometer manufactured by Ion-Tof $\mathrm{GmbH}$ (Münster, Germany). A pulsed sub-nanosecond $\mathrm{Ar}^{+}$ ion beam with a diameter of $50 \mu \mathrm{m}$ and a pulsed current of $0.4 \mathrm{pA}$ was used. The primary ion energy was $10 \mathrm{keV}$. Secondary ions were extracted from the surface by a lens at a potential of $3 \mathrm{keV}$. The ions were mass analyzed by a $1.87-\mathrm{m}$ flight tube that operated in the reflecting mode. A $10-\mathrm{keV}$ postacceleration potential was applied prior to detection of the ions $(\mathrm{KE}=13$ $\mathrm{keV}$ ) by a scintillator-channelplate-photomultiplier combination detector. Spectra were accumulated by a time-to-digital converter in the single-ion counting mode with a maximum of 256 stops per pulse and a time resolution of 472 ps. Data analysis was carried out by using in-house generated software [19].

\section{Sample Preparation}

The initial discovery occurred by using an ether extract of a blood sample obtained from a transplant patient who was undergoing CsA therapy. The blood extract was deposited on an etched $\mathrm{Ag}$ substrate that had been used previously for cocaine analysis [12]. The etched Ag substrates, with varying amounts of cocaine, had been soaking for $\sim 2$ months in a small volume of methanol $(25 \mathrm{~mL})$. These substrates were saved for the purpose of optimizing instrumental conditions for real biological samples. Typically, one blood extract is deposited onto a used substrate to focus and align the ion beams and optimize resolution. This optimization step prevents loss of valuable samples.

In an effort to reproduce the initial finding, $5 \mu \mathrm{L}$ of a cocaine solution $(20 \mu \mathrm{g} / \mathrm{mL})$ was deposited onto etched Ag substrates. Following solvent evaporation, either $2 \mu \mathrm{L}$ of cyclosporin $\mathrm{A}(1 \mu \mathrm{g} / \mathrm{mL}$ in $\mathrm{MeOH})$ or 5 $\mu \mathrm{L}$ of polystyrene $(50 \mu \mathrm{g} / \mathrm{mL}$ in toluene) was deposited on top of the cocaine layer. Note that this sample preparation method differs from the previous work [16-18], which involved premixing of the matrix and analyte. Analysis of the same samples without the addition of cocaine was carried out on Ag substrates etched under identical conditions.

Ion intensity measurements for cyclosporin A used the $\left[{ }^{107} \mathrm{Ag}+\mathrm{CsA}\right]^{+}$corrected peak integral [12]; for polystyrene the integral from $m / z-2200$ to $m / z-$ $3350 \mathrm{u}$ was measured. The corrected peak area refers to a Poisson correction applied to data collected in the single-ion counting mode [20]. This correction function statistically accounts for intensity that is "overlooked" by the detection system as a result of detector dead time. Precision of the measurements is reported as the confidence interval of the mean at $95 \%$ confidence limits $\left(\mathrm{CIM}_{95 \%}\right): \mathrm{CIM}_{95 \%}=x \pm t s / \sqrt{N}$, where, $x$ is the mean, $t$ is the $t$ statistic, $s$ is the standard deviation, and $N$ is the number of determinations; $N$ for all data reported is 6 .

\section{RESULTS}

Before the observed ion intensity enhancement is quantified it is necessary to determine the qualitative 
changes in the TOF-SIMS spectra due to this sample preparation method. The previous work involved analysis of compounds that yielded little or no molecular ion intensity [16-18]. A decrease in fragmentation was observed in these studies. The present work focuses on analysis of compounds that can be detected intact without any matrix sample preparation $[10,11]$. These compounds are lipophilic and tend to cationize well with the substrate.

Figure 1a shows a typical TOF-SIMS spectrum of CsA obtained from an etched Ag substrate with predeposition of cocaine. Cyclosporin A is a lipophilic compound; the amino acid groups of this cyclic peptide are methylated and have over $80 \%$ nonpolar residues. Such lipophilic compounds are cationized preferentially with the substrate (i.e., Ag) in SIMS analysis. This is the case with CsA, with a second ionization pathway cationized with $\mathrm{Na}$ and $\mathrm{K}$, and a slight amount of protonation. The observed isotopic distribution is consistent with other (sA spectra obtained from normal etched $\mathrm{Ag}$ substrates [12], and the [M+ $\mathrm{H}]^{+} /[\mathrm{M}+\mathrm{Ag}]^{+}$intensity ratio does not change statistically when cocaine is predeposited. This indicates that there is no proton donation from the matrix (cocaine) to the analyte (CsA).

Figure $1 \mathrm{~b}$ show a TOF-SIMS spectrum of polystyrene on a cocaine-modified etched $\mathrm{Ag}$ substrate. The $M_{\mathrm{w}}$ was calculated to be $2650 \mathrm{u}$, with a polydispersity index of 1.05 . These values agree with the data provided by the manufacturer and with experimental data obtained from normal substrates. Note that molecular weight determination of low molecular weight polymers by TOF-SIMS correlates well with established methods of molecular weight determination (i.e., gel
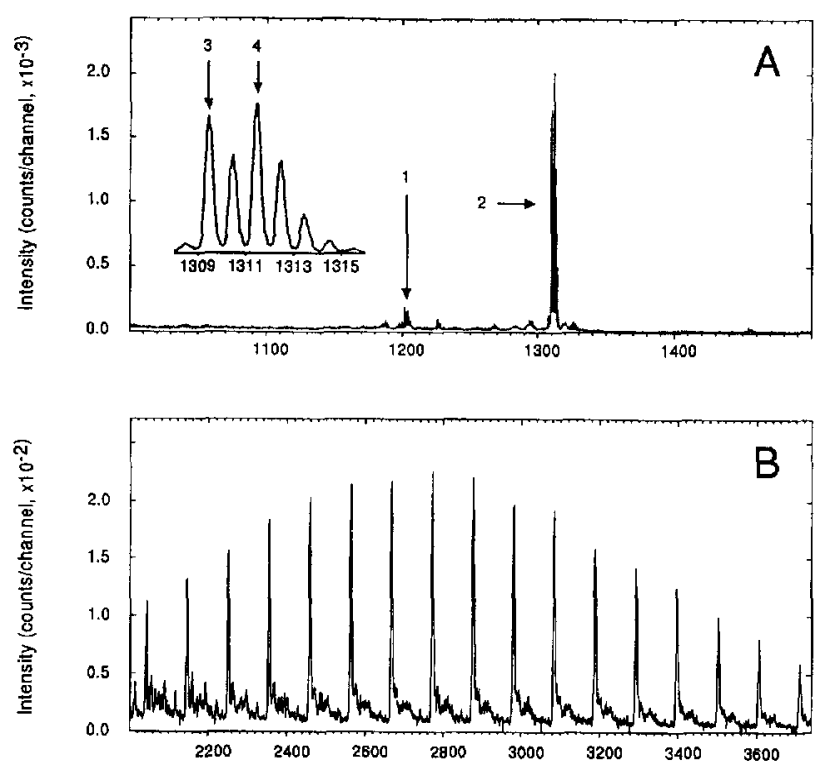

Figure 1. TOF-SIMS spectra obtained from cocaine-modified substrates with (a) cyclosporin $\mathrm{A}$, and $(\mathrm{b})$ polystyrene. The following peaks were noted: $1,[\mathrm{CsA}+\mathrm{H}] ; 2,[\mathrm{CsA}+\mathrm{Ag}]^{\prime} ; 3$, $\left[{ }^{107} \mathrm{Ag}+\mathrm{CsA}\right]^{+}, \mathbf{4},\left[{ }^{109} \mathrm{Ag}+\mathrm{CsA}\right]^{+}$. permeation chromatography and NMR) [21]. Protonated polystyrene is not observed by using either standard or modified preparation techniques. The observed peaks are Ag-cationized, as they are without any matrix preparation [10]. No adducts with cocaine are observed nor does the matrix layer alter the flight times of the polyslyrene (i.e., there are no nonlinear effects). This lack of nonlinear effects indicates that predeposition of a cocaine matrix does not significantly decrease the fragmentation of the polymer nor is there activation of an alternate ionization pathway. Thus, the preparation method does not qualitatively change the TOF-SIMS spectra of these lipophilic polymers and biomolecules. Note that the absolute intensity of cocaine remained constant throughout (only protonated cocaine is observed, at $\mathrm{m} / \mathrm{z} 304$ ).

Table 1 shows the intensity data $\left(\mathrm{CIM}_{95 \%}\right)$ obtained both with and without addition of the cocaine matrix to the surface. Compared to the data from normal (control) substrates, the allograft patient blood extract that contained CsA showed a dramatic increase in ion intensity for the etched Ag substrate that had been soaking in a $\mathrm{MeOH}$ solution that contained cocaine. The increase in SI yield is a factor of $\sim 12$ relative to the control experiment. The intensity data obtained from subsequent experiments that were carried out on a neat CsA sample, in an attempt to reproduce the original finding, also are shown in Table 1. Although the increase in ion intensity was not as dramatic as that from the allograft sample, a reproducible increase was observed. The SI yield was determined to be $\sim 3$ times that of the same sample without cocaine predeposited on the Ag substrate. Comparison of the absolute intensities obtained without the addition of cocaine, shows that the CsA intensity from the allograft sample is about 1500 counts and from the neat sample it is about 3100 . This observation agrees with previous results [11-13] owing to "matrix effects" that decrease SI yield. Extraction techniques (solid phase and ether) do not yield neat samples from blood extracts [12]. Endogenous compounds that are co extracted with the analyte of interest will cause an increase in the surface coverage from blood extracts. This increase in surface coverage in turn causes a decrease in the SI yield of $\mathrm{Ag}$, which results in a decrease in the SI yield of Ag-cationized CsA.

Surprisingly, the CsA intensity from the allograft sample with the addition of cocaine exceeds that of the neat CsA sample of similar concentration with or without cocaine added (Table 1). Also note that the intensity of CsA from the allograft sample is greater than

Table 1. Comparison of intensities $\left(\mathrm{CIM}_{95}\right)$ obtained both with and without addition of cocaine.

\begin{tabular}{lcc}
\hline Sample & Without cocaine & With cocaine \\
\hline \hline Allograft sample & $1,560 \pm 350$ & $18,400 \pm 4,400$ \\
Neat cyclosporin & $3,160 \pm 860$ & $9,560 \pm 2,400$ \\
Neat polystyrene & $144,200 \pm 6,000$ & $407,000 \pm 35,000$ \\
\hline
\end{tabular}


that for the neat solution, both with cocaine added, which may indicate that the cocaine matrix plays a role to decrease the sensitivity of the method to contaminants present in the sample. In the case of real biological fluids, this contamination includes endogenous lipophilic material that has been co-extracted into the ether layer.

In addition, the SI yield obtained from experiments conducted on polystyrene from etched Ag substrates (one that contained a submonolayer of cocaine and the other without) is presented in Table 1. The increase in ion intensity was determined to be a factor of $\sim 2.8$, which is quite similar to that obtained for the neat CsA sample under identical experimental conditions.

\section{DISCUSSION}

As stated earlier, use of the $\mathrm{NH}_{4} \mathrm{Cl}$ matrix reduced fragmentation in analyses of organic ammonium salts and small biomolecules and yielded an increase in the molecular ion intensities of these compounds [16-18]. Busch et al. [18] proposed a gas-phase mechanism by which the matrix $\left(\mathrm{NH}_{4}^{+}\right.$ions) and the analyte are sputtered in large clusters; the "desolvation" process of the matrix from the analyte is responsible for relaxation of the internal energy of the analyte, which results in reduced fragmentation. The present work does not report any decrease in fragmentation that results from use of the cocaine matrix. However, it is not known for certain whether use of the cocaine matrix does, indeed, yield a reduction in fragmentation for other analytes. If, in the future, the method does prove to decrease fragmentation, it would be advantageous for drug and metabolite studies to ensure that fragmentation products are not mistaken for metabolites.

Unger and co-workers [16] emphasized acid-base properties by which proton transfer would take place from the matrix to the analyte. This transfer is unlikely, however, in the case when cocaine is used as a matrix. Cocaine is basic and possesses a tertiary amine with a $\mathrm{p} K_{a}$ of approximately 10 . The proton affinity for a tertiary amine is $\sim 225 \mathrm{kcal} / \mathrm{mol}$. This high value accounts for the extraordinary SI yield observed for cocaine-a preformed cation (protonated molecular ion). Thus, a gas-phase proton transfer is not likely to occur from cocaine to either cyclosporin A or polystyrene. This conclusion is supported experimentally because the $[\mathrm{M}+\mathrm{H}]^{+} /[\mathrm{M}+\mathrm{Ag}]^{+}$ratio does not change for either compound and the absolute intensity of cocaine does not change when a sample overlayer is applied. Thus, because acid-base reactions are expected to play a role in SI yield enhancement and because the matrix is predeposited as opposed to mixed with the analyte (i.e., the matrix most likely does not solvate the analyte), different mechanisms must be considered.
Three mechanisms can be suggested based on previous studies [10] of the structure of polymers deposited on etched $\mathrm{Ag}$ substrates. The first proposed mechanism is illustrated in Figure 2a. A submonolayer of cocaine may act as an energy transfer medium between the $\mathrm{Ag}$ and the analyte. The collisional cascade induced by the impinging $\mathrm{Ar}^{+}$ion transfers energy to surface $\mathrm{Ag}$ atoms (i.e., energy deposited by prirrlary ions to the surface exceeds the Ag lattice energy). Subsequent energy transfer from $\mathrm{Ag}$ results in desorption of the analyte material that resides on the surface. The cocaine layer may absorb the energy of the $\mathrm{Ag}$ atoms and distribute it over a larger area to assist in desorption of larger species. Also, if the analyte material resides above the cocaine layer as shown, the binding energy of the analyte (e.g., CsA) to the "cocaine surface" would be smaller than the binding energy to the silver, which facilitates desorption.

Figure $2 b$ illustrates an alternative mechanism where the cocaine plays a role by "blocking" sites on the Ag substrate. This mechanism assumes that the cocaine specifically orients itself on the surface (i.e., the phenyl ring interacts with the $\mathrm{Ag}$ ). The result of this surface configuration would be that the positively charged tertiary amine residue on the cocaine directs lipophilic compounds (e.g., CsA, polystyrene) to Ag sites that are unoccupied by cocaine (i.e., the deposited analyte material distributes on the surface where no cocaine resides). This mechanism would provide a means to homogeneously reduce the surface coverage that results from the deposited analyte. Because cocaine is a preformed cation, it is easy to desorb from the surface. In this case, if cocaine lies on top of the Ag where no analyte resides, these $\mathrm{Ag}$ sites will be exposed more readily, which thus allows $A g$ to reach the gas phase and ionize the analyte.

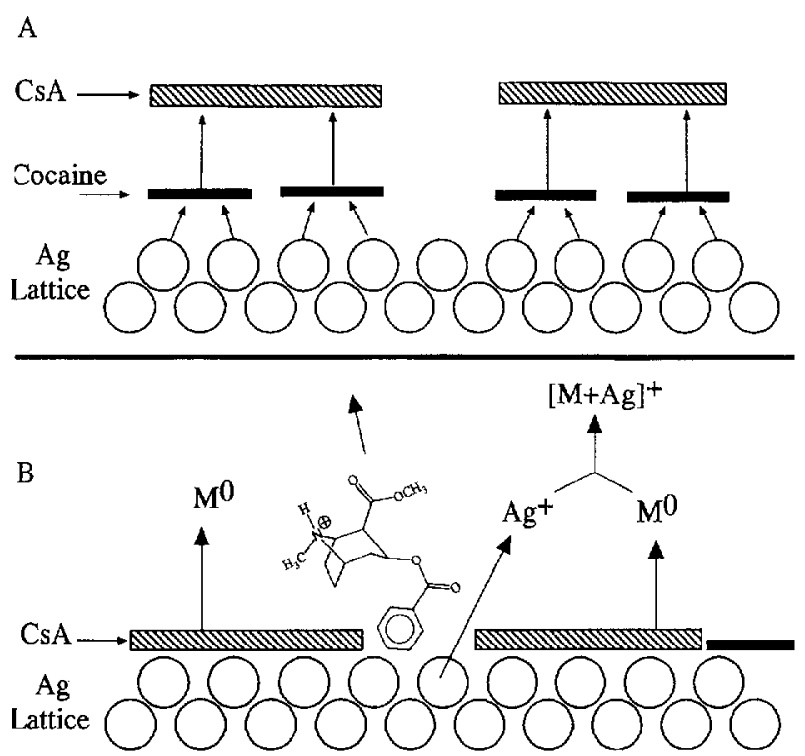

Figure 2. (a) Energy-transfer/reduced surface-binding mechanism. (b) Surface coverage mechanism. 
The simplest explanation may arise from the higher surface area of etched $\mathrm{Ag}$ substrates relative to nonetched Ag. Previously it was shown that an etched Ag substrate can hold almost 3 orders of magnitude more polymer than expected based on geometric surface coverage measurements [10]. Thus, the cocaine may occupy nonactive SIMS sites (i.e., deep inside the pores where the ion beam does not sample). When the analyte material is deposited, a higher proportion of the analyte is forced to reside on top of the surface, which is accessible by the ion beam.

Regardless of the mechanism involved, our results indicate that SI yield can be enhanced by predepositing a submonolayer of cocaine on an etched Ag substrate. The high SI yield of cocaine is attributed to the fact that cocaine is a preformed cation. Because ammonium chloride matrices also provide a significant abundance of preformed cations $\left(\mathrm{NH}_{4}^{+}\right)$, preformed cations could be the molecules most useful to increase SI yield in SIMS. Other molecules that give high SI yield in the TOF-SIMS and therefore also may be good candidates for matrices are rhodamine- $6 \mathrm{G}$, flurazepam, and chlorpromazine, to name a few.

Obviously further experiments are necessary to elucidate the mechanism of this effect. These investigations include the use of other performed cations, determination of dependency on molecular weight of the predeposited species, and characterization of the surface structure. It is also important to determine if the new preparation technique retains the quantitative abilities of the TOF-SIMS for analysis of biomolecules. However, the reproduction of the initial observation through iterative experiments is the most logical next step; this may include soaking etched $\mathrm{Ag}$ targets in varying concentrations of cocaine for extended periods of time ( $\sim 2$ months).

The results show that this method of sample preparation is successful to increase the SI yield of materials that do not typically give high SI yield (e.g., analysis from physiological media), as well as those that tend to cationize with the substrate (c.g., Ag-cationized). These materials include higher molecular weight biologically important compounds, analysis from blood extracts, and polymer samples. If the enhancement of the SI yield of biomolecules and polymers in SIMS can be achieved routinely by using this method (or a modified method), several attractive applications are made possible. For example, the increase in ion intensity translates into lower detection limits that are important in the analysis of drugs in real biological samples. The immunosuppressant FK-506 has low therapeutic blood levels $(0.5-60 \mathrm{ng} / \mathrm{mL})$ and this method will allow access to such a low concentration range. As previously stated, the preparation technique appears to decrease the sensitivity of TOF-SIMS to endogenous material. This decreased sensitivity may allow elimination of some extraction steps in the analysis of biological fluids. Furthermore, if the mechanism lies in the desorption process (i.e., mechanism in Figure 2a), the method may allow extension of the mass region for polymers and biopolymers where interaction of the analyte with the surface (i.e., high surface binding due to a large number of weak bonds) limits the mass range.

Further elucidation of the mechanism should allow the initial results to be superseded. Now that new biological applications are under development within the mass range of SIMS, a new preparation method to provide better detection limits and simplify the purification process for real biological fluids would be beneficial. Development of this sample preparation method could facilitate routine biomedical analysis by time-of-flight SIMS.

\section{Acknowledgments}

This work was supported by the National Science Foundation under Grant CHE-9022135 and by the U.S. Environmental Protection Agency under Grant R819809-01. The authors wish to thank the reviewers for their helpful comments and suggestions.

\section{References}

1. Bletsos, I. V.; Hercules, D. M.; van Leyen, D; Hagenhoff, B.; Niehuis, E.; Benninghoven, A. Anal. Chem. 1991, 60, 938.

2. Bletsos, I. V.; Hercules, D. M.; van I eyen, D.; Benninghoven, A.; Karakatsanis, C. G.; Rieck, J. N. Macromolecules 1990, 23, 4157.

3. van Leyen, D.; Hagenhoff, B.; Niehuis, E.; Benninghoven, A.; Bletsos, I. V.; Hercules, D. M. I. Vac. Sci. Technol. A. 1989, 7, 1790.

4. Davies, M. C.; Lynn, R. A. P; Davis, S. S.; Hearn, J.; Vickerman, J. C.; Paul, A. J. J. Colloid Interface Sci. 1993, 161, 83-90.

5. Bletsos, I. V.; Hercules, D. M.; Dieter, V. L.; Hagenhoff, B.; Niehuis, E.; Benninghoven, A. Anal. Chem. 1991, 63, 83-90.

6. Linton, R. W.; Mawn, M. P.; Belu, A. M. DeSimone, J. M.; Hunt, M. O., Jr.; Menceloglu, Y. Z.; Cramer, H. G.; Benninghoven, A. Surf. Interface Anal. 1993, 20, 991-999.

7. Treverton, J. A.; Paul, A. J.; Vickerman, J. C. Surf. Interface Anal. 1993, 20, 449-456.

8. Bennett, J.; Dagata, J. A. J. Vac. Sci. Technol. B, 1994, 12, 214-18.

9. Lafortune, F.; Buchko, G. W.; Hruska, F. E.; Sadana, K. L.; Standing, K. G.; Westmore, J. B. Nucleosides Nucleotides 1992, 11, 1305-1324.

10. Muddiman, D. C.; Brockman, A. H.; Proctor, A.; Houalla, M.; Hercules, D. M. J. Phys. Chem. 1994, 98, 11570-11575.

11. Muddiman, D. C.; Gusev, A. I.; Proctor, A.; I Iercules, D. M.; Venkataramanan, R.; Diven, W. Anal. Chem. 1994, 66, 2362-2368.

12. Muddiman, D. C.; Nicola, A. J.; Proctor, A.; Hercules, D. M. Appl. Spectrosc. 1995, in press.

13. Muddiman, D. C.; Gusev, A. I.; Martin, L. B.; Hercules, D. M. Fresenius' J. Anal. Chem. 1995, in press.

14. Muddiman, D. C.; Gusev, A. I.; Stoppek-Langner, K.; Proctor, A.; Hercules, D. M.; Tata, P.; Venkataramanan, R.; Diven, W. J. Mass Spectrom. 1995, 30, 1469-1479.

15. Seedorf, U. Fobker, M.; Voss, R.; Meyer, K.; Kannenberg, F.; Meschede, D.; Ullrich, K.; Horst, J.; Benninghoven, A.; Assmann, G. Clin. Chem. 1995, 41, 548. 
16. Liu, L. K.; Busch, K. L.; Cooks, R. G. Anal. Chem. 1981, 53, 109-113.

17. Unger, S. E.; Day, R. J.; Cooks, R. G. Int. J. Mass Spectrom. Ion Phys. 1981, 39, 231-255.

18. Bussh, K. I.; Hsu, R. H.; Xje, Y. X.; Cooks, R. G. Anal. Chem. 1983, 55, 1157-1160.

19. Proctor, A. GOOGLY Software, Copyright 1994, All rights reserved.
20. Muddiman, D. C.; Nicola, A. J.; Proctor, A.; Hercules, D. M. Proceedings of the 43rd ASMS Conference on Mass Spectrometry and Allied Topics; Atlanta, GA; American Society for Mass Spectrometry: Santa Fe, NM, 1995; p 996.

21. Hittle, L. R.; Hercules, D. M. Surf. Interface Anal. 1994, 21, $217-225$. 